\title{
Genetic risk factors and retinal ganglion cell degeneration in primary open-angle glaucoma (POAG): A bird's eye view
}

\author{
Barkur S. Shastry \\ Department of Biological Sciences, Oakland University, Rochester, USA \\ Email: barkurshastry@yahoo.com
}

Received 1 April 2013; revised 3 May 2013; accepted 20 May 2013

Copyright (C) 2013 Barkur S. Shastry. This is an open access article distributed under the Creative Commons Attribution License, which permits unrestricted use, distribution, and reproduction in any medium, provided the original work is properly cited.

\begin{abstract}
Glaucoma is an optic neuropathy and often associated with elevated intraocular pressure (IOP). It is the second leading cause of irreversible blindness worldwide and is characterized by the optic nerve degeneration and loss of retinal ganglion cells (RGCs). This may lead to loss of vision. The primary cause of glaucoma is unknown but several risk factors including elevated IOP and age have been suggested. In most population, primary open-angle glaucoma (POAG) is the most common type of glaucoma and is often associated with elevated IOP. Genetic analyses have identified at least 14 chromosomal loci but only three genes which when mutated can cause POAG have been well documented. These genes account for less than $5 \%$ of all POAG cases suggesting that more than $90 \%$ of the genetic contribution of POAG cases is unknown. RGC consists of cell body, axon and dendritic arbor and each of these three parts can independently degenerate. Several molecular signals such as oxidative stress, mitochondrial dysfunction, disruption of neurotrophic factor (NTF), dysfunction of immune system, glial activation and the release of tumor necrosis factor (TNF) have been found to be involved in the optic nerve degeneration. Therefore, therapies aimed at axonal and cell body protection may have a greater protective role in early or progressive glaucoma. In the future, an understanding of gene-gene and geneenvironmental factor interaction as well as epigenetic regulation of gene expression by environmental factors may provide an opportunity to develop neuroprotective therapies and DNA based diagnostic tests.
\end{abstract}

Keywords: Degeneration, Gene, Mutation, Retina

\section{INTRODUCTION}

Glaucoma is clinically and genetically complex neurodegenerative disorder of the eye. It is the second lead- ing cause of blindness affecting approximately 70 million people worldwide [1]. The condition affects all age groups throughout the world and is classified into primary open-angle (POAG), primary angle-closure (PACG) and primary congenital (PCG) glaucoma [2-5]. The disorder is clinically characterized by the progressive degeneration of the retinal ganglion cells (RGCs) and is frequently associated with elevated intraocular pressure (IOP) that leads to optic nerve damage and irreversible loss of vision. Previous studies suggest that glaucomatous neuropathy is associated with several molecular signals such as oxidative stress, disruption of neurotrophic factor (NTF), dysregulation of immune system, glial activation, mitochondrial dysfunction and the release of tumor necrosis factor (TNF) [6]. Epidemiological studies suggest that POAG is the most common type of glaucoma in most populations and is consistently associated with elevated IOP $[7,8]$. However, patients with POAG can also have an IOP within the normal range and they are classified as having normal tension glaucoma (NTG) —most likely a different entity [4]. In addition, in some patients without elevated IOP, optic neuropathy develops [9]. Therefore, elevation in IOP is neither necessary nor sufficient for the onset or progression of the disorder. This clinical heterogeneity may also indicate genetic heterogeneity of the disease. It is likely that genetic, epigenetic and environmental factors may influence the development of POAG [10].

\section{GENETIC AND ENVIRONMENTAL FACTORS}

The mode of inheritance of glaucoma is unclear. However, twin studies and the prevalence of the disorder in first-degree relatives suggest genetic contribution to the development of glaucoma [11,12]. Accordingly, genetic analyses have identified at least 14 chromosomal loci [2, 13,14] for POAG but only three genes (myocilin, optineurin and WDR 36) which when mutated can cause 
POAG have been well documented. These three genes account for less than $5 \%$ of all POAG cases $[14,15]$ suggesting that more than $90 \%$ of the genetic contribution of POAG cases is unknown. Recently, a significant association of two common variants in an intergenic region between the CAV1 and CAV2 (encoding caveolin 1 and 2) $[16,17]$ and SNPs located near the TMCO1 (transmembrane and coiled-coil domain-1) and CDKN2B-AS [18, 19] genes in POAG as well as in NTG (CDKN2BAS) have been reported. However, the association of CAV1 and CAV2 has not been replicated in other populations $[20,21]$ suggesting that this risk factor may not have a major role in all populations. The CDKN2B-AS (cyclin dependent kinase inhibitor) gene product is involved in the regulation of expression of CDKN2B gene which is a component of transforming growth factor (TGF-beta) signaling pathway. This pathway was previously shown to be involved in glaucoma [22]. Although the exact role of myocilin (MYOC), optineurin (OPTN) and WDR 36 (tryptophan and aspartic acid repeat domain 36) genes in the pathogenesis of glaucoma is unknown, the biology of mutant genes has been studied and discussed previously $[4,5,15]$. For instance, mutations in MYOC gene is reported to sensitize cells to oxidative stress induced apoptosis [23], mitochondrial defects [24] that may lead to trabecular meshwork cell death and endoplasmic reticulum stress [25] that may cause cellular toxicity and death. Similarly, mutation in OPTN gene (E50K) selectively induced the death of RGCs and this was inhibited by antioxidants suggesting that cell death is mediated by oxidative stress [26]. This may have implications for the pathogenesis. In the same way, mutations in the WDR 36 gene may interfere in a variety of cellular processes such as signal transduction that may lead to cell death. Interestingly, inherited glaucoma in animals however, does not involve mutations in MYOC, CYP1B1-a member of the cytochrome P450 enzymes encoding genes-and neurotrophic factor 4 (NTF4) genes [27-30].

Although hereditary components of PACG exist, causative genes have not been identified except occasional difference in the frequency of polymorphisms in some genes [3]. PCG is an important cause of visual loss in children and the familial condition is inherited as an autosomal recessive trait. More than 60 different mutations in CYP1B1 (or GLC3A) have been reported in several PCG families [5]. NTG is relatively a less explored avenue of research and there has been paucity of research into the genetic basis of NTG [4]. In addition to genetic predisposition, environmental factors such as drinking coffee, wearing tight neckties, smoking, dietary fat, postmenopausal hormone use and other life-style factors may influence the development of glaucoma by affecting IOP and RGCs death [31]. One example to illustrate this is the pseudoexfoliation syndrome that is the most common subtype of POAG. This disorder is shown to be associ- ated with mutations in lysyl oxidase like-1 (LOXL1) gene in addition to geographic and climatic factors such as sun exposure and ambient temperature [32]. Similarly, epigenetic regulation of gene expression by environmental factors may also play a role in the development of glaucoma. For instance, in experimental glaucoma models, it has been found that several ganglion cell marker genes are down regulated in RGCs [33-37]. This may lead to an early onset atrophy of ganglion cells [38]. In addition, the pathologic course of RGC involves histone modification [39] and histone deacetylase 4 (HDAC4) may have a role in the survival of retinal neurons $[40,41]$. Similarly, histone acetyltransferase p300 was found to promote intrinsic axonal regeneration [42].

\section{RGC DEGENERATION}

Retinal ganglion cell consists of cell body, axon and dendritic arbor and each of these three parts can independently degenerate. The mechanism of RGC death is not well understood. A current proposal based on the localized retinal nerve fiber layer defects in glaucoma patients suggests that the axon is the first one to be affected by the elevated IOP $[6,43,44]$. According to rodent models also axon dysfunction and degeneration are early events in RGCs death $[45,46]$. This axonal degeneration could be due to lack of support function from astrocytes that are the major resident cell population of axons in addition to lamina cribrosacytes. Once the axonal function is lost it cannot transport neurotrophic input to cell bodies and in absence of neurotrophic input RGC bodies may be activated for degeneration by a distinct activation pathway [47]. This may lead to ganglion cells to undergo apoptosis program by the activation of several kinases and caspase. However, it is not clear how these kinases are activated and how axonal damage is transmitted to the cell soma. The mechanism of degeneration of axons and neuronal cell bodies may also differ from one another [6]. It is likely that ganglion cells are initially damaged at the level of optic nerve head and the secondary degeneration involving somatic components damage the surrounding healthy ganglion cells. Alternatively the dying ganglion cells may affect other cells such as microglia, macroglia and Muller cells. These cells secrete several other molecules such as cytokines that may lead to the activation of extrinsic apoptosis program. However, it is not clear whether the secondary degeneration in glaucoma occurs or not. A more comprehensive treatment of this subject was appeared previously [43].

There are several molecular signals such as oxidative stress, dysfunction of immune system, retrograde transport of neurotrophic factor, glial activation, release of tumor necrosis factor (TNF) and mitochondrial dysfunction that are associated with ganglion cell degeneration $[6,43,44]$. These signals may converge to induce RGC death. For instance, in some patients, IOP elevation may 
contribute to specific changes in optic nerve that may lead to axonal degeneration and subsequent RGC death [43]. In addition, defective retrograde axonal transport of neurotrophic factors and disruption of the axonal motor protein expression may contribute to the degeneration $[48,49]$. Glial cell activation produces tumor necrosis factor (TNF) alpha and interleukin (IL) beta as major cytokines. These are involved in several pathways and may mediate RGC death [50,51]. Recent report also suggests that neurodegenerative and inflammatory pathway components are linked to TNF-alpha/TNFR1 signaling in the glaucomatous retina [52]. Increase in endoplasmic reticulum (ER) stress may induce the accumulation of miss-folded proteins and that may result in the induction of RGC degeneration by apoptosis [53]. Similarly, oxidative stress may play an important role in retinal neuronal degeneration. It induces severe damage to cellular proteins, nucleic acids, mitochondria and subsequently can lead to cell body death [54,55]. Mitochondrial dysfunction due to oxidative injury has also been reported in RGC body death [56]. Several types of stress including IOP also activate endothelin-1 receptors such as ETRA and ETRB in the optic nerve and retina. This may be subsequently involved in RGC body death by ischemia and apoptosis [57-59]. Similarly, excitotoxicity by glutamate may be responsible for optic neuropathy through the stimulation of NMDA receptor that may initiate several molecular pathways such as increase in intracellular calcium influx, activation of apoptotic pathway and initiation of inflammation [60-62]. Thus, it appears that these varieties of signals converge to produce the glaucoma pathology. Recently, aggregation and deformability of erythrocytes have been reported in POAG [63] suggesting decreased blood flow to the optic nerve.

\section{CONCLUDING REMARKS}

Glaucoma is a complex neurodegenerative disorder of the eye. Several factors such as genetic, environmental and epigenetic contribute to the degenerative processes of the retina. The progressive nature of the disease may also support the idea about the accumulation of several insults that ultimately results in glaucoma pathology. In the future, it is necessary to have neuroprotection studies and we need to understand how degeneration of one part of the neuron affects another part. In addition, we need to know the epigenetic changes such as promoter methylation, activation of histone deacetylases and deacetylation of histones occurring in the nucleus [64]. Therapies aimed at axonal and cell body protection may have a greater protective role in early or progressive glaucoma.

\section{REFERENCES}

[1] Quigley, H. (1996) Number of people with glaucoma worldwide. British Journal of Ophthalmology, 80, 389393. doi:10.1136/bjo.80.5.389

[2] Shastry, B.S. (2011) Genetic risk factors in glaucoma. In: Berhardt, L.E. Ed., Advances in Medicine and Biology, Nova Science Publishers, Inc., New York, 71-87.

[3] Shastry, B.S. (2013) Genetic susceptibility to primary angle-closure glaucoma. Discovery Medicine, 15, 17-22.

[4] Shastry, B.S. (2013) Genetic susceptibility to normal tension glaucoma. British Journal of Medicine and Medical Research, 3, 372-382.

[5] Shastry, B.S. (2013) Emerging concept of genetic and epigenetic contributions to the manifestation of glaucoma. In: Rumelt, S., Ed., Glaucoma: Basic and Clinical Aspects, InTech Inc., Israel, in press.

[6] Munemasa, Y. and Kitaoka, Y. (2012) Molecular mechanisms of retinal ganglion cell degeneration in glaucoma and future prospects for cell body and axonal protection. Frontiers in Cellular Neuroscience, 6, 60-72.

[7] Quigley, H. (1993) Open-angle glaucoma. New England Journal of Medicine, 328, 1097-1106. doi:10.1056/NEJM199304153281507

[8] Fuse, N. (2010) Genetic bases of glaucoma. Tohoku Journal of Experimental Medicine, 221, 1-10. doi:10.1620/tjem.221.1

[9] Anderson, D.R., Drance, S.M. and Schulzer, M. (2001) Natural history of normal tension glaucoma. Ophthalmology, 108, 247-253. doi:10.1016/S0161-6420(00)00518-2

[10] Wiggs, J.L. (2012) The cell and molecular biology of complex forms of glaucoma: updates on genetic, environmental and epigenetic risk factors. Investigative Ophthalmology \& Visual Science, 53, 2467-2469. doi:10.1167/iovs.12-9483e

[11] Gottfredsdottir, M.S., Sverrisson, T., Musch, D.C., et al. (1999) Chronic open-angle glaucoma and associated ophthalmic findings in monozygotic twins and their spouses in Iceland. Journal of Glaucoma, 8, 134-139. doi:10.1097/00061198-199904000-00009

[12] Teikari, J.M. (1990) Genetic influences in open-angle glaucoma. International Ophthalmology Clinic, 30, 161168. doi:10.1097/00004397-199030030-00003

[13] Liu, Y. and Allingham, R.R. (2011) Molecular genetics in glaucoma. Experimental Eye Research, 93, 331-339. doi:10.1016/j.exer.2011.08.007

[14] Fingert, J.H. (2011) Primary open-angle glaucoma genes. Eye, 25, 587-595. doi:10.1038/eye.2011.97

[15] Fingert, J.H., Stone, E.M., Sheffield, V.C., et al. (2002) Myocilin glaucoma. Survey of Ophthalmology, 47, 547561. doi:10.1016/S0039-6257(02)00353-3

[16] Thorleifsson, G., Walters, G.B., Hewitt, A.W., et al. (2010) Common variants near CAV1 and CAV2 are associated with primary open-angle glaucoma. Nature Genetics, 42 , 906-909. doi:10.1038/ng.661

[17] Wiggs, J.L., Kang, J.H., Yaspan, B.L., et al. (2011) Common variants near CAV1 and CAV2 are associated with primary open-angle glaucoma in Caucasian from the United States. Human Molecular Genetics, 20, 4707-4713. doi:10.1093/hmg/ddr382 
[18] Burdon, K.P., Macgregor, S., Hewitt, A.W., et al. (2011) Genome wide association study identifies susceptibility loci for open-angle glaucoma at TMCO1 and CDKN2BAS1. Nature Genetics, 43, 574-578. doi:10.1038/ng.824

[19] Wiggs, J.L., Yaspan, B.L., Hauser, M.A., et al. (2012) common variants at 9p21 and 8q22 are associated with increased susceptibility to optic nerve degeneration in glaucoma. Public Library of Science Genetics, 8, e1002654.

[20] Abo-Amero, K.K. Kondkar, A.A., Mousa, A., et al. (2012) Lack of association of SNP rs 4236601 near CAV1 and CAV2 with POAG in a Saudi cohort. Molecular Vision, 18, 1960-1965.

[21] Kuehn, M.H., Wang, K., Roos, B., et al. (2011) Chromosome 7q31 POAG locus: ocular expression of caveolins and lack of association with POAG in a US cohort. Molecular Vision, 17, 430-435.

[22] Sethi, A., Mao, W., Wordinger, R.J., et al. (2011) Transforming growth factor beta induces extracellular matrix protein cross-linking lysyl oxidase (LOX) gene in human trabecular meshwork cells. Investigative Ophthalmology \& Visual Science, 52, 5240-5250. doi:10.1167/iovs.11-7287

[23] Joe, M.K. and Tomarrev, S.I. (2010) Expression of myocilin mutant sensitizes cells to oxidative stress induced apoptosis: Implications to glaucoma pathogenesis. American Journal of Pathology, 176, 2880-2890. doi:10.2353/ajpath.2010.090853

[24] He, Y., Leung, K.W., Zhou, Y.H., et al. (2009) Pro370Leu mutant myocilin impairs mitochondrial function in human trabecular meshwork cells. Molecular Vision, 15, 815-825.

[25] Joe, M.K., Sohn, S., Hur, W., et al. (2003) Accumulation of mutant myocilins in ER leads to ER stress and potential cytotoxicity in human trabecular meshwork cells. Biochemical Biophysical Research Communication, 312, 592-600. doi:10.1016/j.bbrc.2003.10.162

[26] Chalasani, M.L., Radha, V., Gupta, V., et al. (2007) A glaucoma associated mutant optineurin selectively induces death of retinal ganglion cells which is inhibited by antioxidants. Investigative Ophthalmology \& Visual Science, 48, 1607-1614. doi:10.1167/iovs.06-0834

[27] Kato, K., Sasaki, N., Matsunaga, S., et al. (2007) Cloning of canine myocilin cDNA and molecular analysis of the myocilin gene in Shiba Inu dogs. Veterinary Ophthalmology, 10, 53-62. doi:10.1111/j.1463-5224.2007.00530.x

[28] Kato, K., Kamida, A., Sasaki, N., et al. (2009) Evaluation of the CYP1B1 gene as a candidate gene in beagles with open-angle glaucoma. Molecular Vision, 15, 2470-2474.

[29] Kato, K., Sasaki, N., Gelatt, K.N., et al. (2009) Autosomal recessive primary open-angle glaucoma (POAG) in beagles is not associated with mutations in myocilin (MYOC) gene. Graefes Archives of Clinical and Experiments Ophthalmology, 247, 1435-1436. doi:10.1007/s00417-009-1053-2

[30] Kato, K., Sasaki, N. and Shastry, B.S. (2012) Retinal ganglion cell (RGC) death in glaucomatous beagles is not associated with mutations in p53 and NTF4 genes. Veterinary Ophthalmology, 15, 8-12.
doi:10.1111/j.1463-5224.2012.01024.X

[31] Kang, J.H., Wiggs, J.L., Rosener, B.A., et al. (2010) Endothelial nitric oxide synthase gene variants and primary open-angle glaucoma: interaction with sex and postmenopausal hormone use. Investigative Ophthalmology \& Visual Science, 51, 971-979. doi:10.1167/iovs.09-4266

[32] Stein, J.D., Pasguale, L.R. and Talwar, N. (2011) Geographic and climatic factors associated with exfoliation syndrome. Archives of Ophthalmology, 129, 1053-1060. doi:10.1001/archophthalmol.2011.191

[33] Ahmed, F., Brown, K.M., Stephan, D.A., et al. (2004) Microarray analysis of changes in mRNA levels in the rat retina after experimental elevation of intraocular pressure. Investigative Ophthalmology \& Visual Science, 45, 12471258. doi:10.1167/iovs.03-1123

[34] Huang, W., Fileta, J., Guo, Y. and Grosskreutz, C.L. (2006) Down regulation of Thy1 in retinal ganglion cells in experimental glaucoma. Current Eye Research, 31, 265271.

[35] Weishaupt, J.H., Klocker, N. and Bahr, M. (2005) Axotomy induced early down regulation of POU-IV class transcription factors Brn-3a and Brn-3b in retinal ganglion cells. Journal of Molecular Neuroscience, 26, 17-25. doi:10.1385/JMN:26:1:017

[36] Yan, Z., Qugley, H.A., Pease, M.E., et al. (2007) Changes in gene expression in experimental glaucoma and optic nerve transection: The equilibrium between protective and detrimental mechanisms. Investigative Ophthalmology \& Visual Science, 48, 5539-5548. doi:10.1167/iovs.07-0542

[37] Soto, I., Oglesby, E., Buckingham, B.P., et al. (2008) Retinal ganglion cells down regulate gene expression and lose their axons within the optic nerve head in a mouse glaucoma model. Journal of Neuroscience, 28, 548-561. doi:10.1523/JNEUROSCI.3714-07.2008

[38] Morgan, J.E. (2002) Retinal ganglion cell shrinkage in glaucoma. Journal of Glaucoma, 11, 365-370. doi:10.1097/00061198-200208000-00015

[39] Pelzel, H.R., Schlamp, C.L. and Nickells, R.W. (2010) Histone $\mathrm{H} 4$ deacetylation plays a critical role in early gene silencing during neuronal apoptosis. Biomedical Central Neuroscience, 11, 62. doi:10.1186/1471-2202-11-62

[40] Chen, B. and Cepko, C.L. (2007) Requirement of histone deacetylase activity for the expression of critical photoreceptor gene. Biomedical Central Developmental Biology, 7, 78 .

[41] Chen, B. and Cepko, C.L. (2009) HDAC4 regulates normal survival in normal and diseased retinas. Science, 323, 256-259. doi:10.1126/science.1166226

[42] Gaub, P., Joshi, Y., Wuttke, A., et al. (2011) The histone acetyltransferase p300 promotes intrinsic axonal regeneration. Brain, 134, 2134-2148. doi:10.1093/brain/awr142

[43] Nickells, R.W. (2012) The cell and molecular biology of glaucoma: Mechanisms of retinal ganglion cell death. Investigative Ophthalmology \& Visual Science, 53, 24762481. 
[44] Almasieh, M., Wilson, A.M., Morquette, B., et al. (2012) The molecular basis of retinal ganglion cell death in glaucoma. Progress in Retinal and Eye Research, 31, 152-181. doi:10.1016/j.preteyeres.2011.11.002

[45] Schlamp, C.L., Li, Y., Dietz, J.A., et al. (2006) Progressive ganglion cell loss and optic nerve degeneration in $\mathrm{DBA} / 2 \mathrm{~J}$ mice is variable and asymmetric. Biomedical Central Neuroscience, 7, 66. doi:10.1186/1471-2202-7-66

[46] Howell, G.R., Libby, R.T., Jacobs, T.C., et al. (2007) Axons and retinal ganglion cells are insulted in the optic nerve early in DBA/2J glaucoma. Journal of Cell Biology, 179, 1523-1537. doi:10.1083/jcb.200706181

[47] Harder, J.M. and Libby, R.T. (2011) BBC3 (PUMA) regulates developmental apoptosis but not axonal injury induced death in the retina. Molecular Neurodegeneration, 6, 50. doi:10.1186/1750-1326-6-50

[48] Morrison, J.C., Dormann-Pease, M.E., Dunkelberger, G.R., et al. (1990) Optic nerve head extracellular matrix in primary optic atrophy and experimental glaucoma. Archives of Ophthalmology, 108, 1020-1024. doi:10.1001/archopht.1990.01070090122053

[49] Martin, K.R., Quigley, H., Valenta, D., et al. (2006) Optic nerve dynein motor protein distribution changes with intraocular pressure elevation in a rat model of glaucoma. Experimental Eye Research, 83, 255-262. doi:10.1016/j.exer.2005.11.025

[50] Tezel, G. and Wax, M.B. (2000) Increased production of tumor necrosis factor alpha by glial cell exposed to stimulated ischemia or elevated hydrostatic pressure induced apoptosis in cultured retinal ganglion cells. Journal of Neuroscience, 20, 8693-8700.

[51] Kitaoka, Y., Kitaoka, Y., Kwong, J.M., et al. (2006) TNF-alpha induced optic nerve degeneration and nuclear factor kappa Bp65. Investigative Ophthalmology \& Visual Science, 47, 1448-1457. doi:10.1167/iovs.05-0299

[52] Yang, X., Luo, C., Cai, J., et al. (2011) Neurodegenerative and inflammatory pathway components linked to TNF-alpha/TNFR ${ }_{1}$ signaling in the glaucomatous human retina. Investigative Ophthalmology \& Visual Science, 52, 8442-8454. doi:10.1167/iovs.11-8152

[53] Doh, S.H., Kim, J.H., Lee, K.M., et al. (2010) Retinal ganglion cell death induced by endoplasmic reticulum stress in a chronic glaucoma model. Brain Research, 1308, 158-166. doi:10.1016/j.brainres.2009.10.025

[54] Tezel, G. (2006) Oxidative stress in glaucomatous neurodegeneration: Mechanisms and consequences. Progress in Retinal and Eye Research, 25, 490-513. doi:10.1016/j.preteyeres.2006.07.003

[55] Yuki, K., Ozawa, Y., Yoshida, T., et al. (2011) Retinal ganglion cell loss in superoxide dismutase 1 deficiency. Investigative Ophthalmology \& Visual Science, 52, 41434150. doi:10.1167/iovs.10-6294

[56] Lieven, C.J., Schlieve, C.R., Hoegger, M.J., et al. (2006) Retinal ganglion cell axotomy induces an increase in intracellular superoxide anion. Investigative Ophthalmology \& Visual Science, 47, 1477-1485. doi:10.1167/iovs.05-0921

[57] Minton, A.Z., Phatak, N.R., Stankowska, D.L., et al. (2012) Endothelin B receptors contribute to retinal ganglion cell loss in a rat model of glaucoma. Public Library of Science One, 7, e43199.

[58] Tonari, M., Kurimoto, T., Horie, T., et al. (2012) Blocking endothelin B receptor rescues retinal ganglion cells from optic nerve injury through suppression of neuroinflammation. Investigative Ophthalmology \& Visual Science, 53, 3490-3500. doi:10.1167/iovs.11-9415

[59] Lau, J., Dang, M., Hockmann, K., et al. (2006) Effects of acute delivery of endothelin-1 on retinal ganglion cell loss in the rat. Experimental Eye Research, 82, 132-145. doi:10.1016/j.exer.2005.06.002

[60] Dong, C.J., Guo, Y., Agey, P., et al. (2008) Alpha 2 adrenergic modulation of NMDA receptor function as a major mechanism of RGC protection in experimental glaucoma and retinal excitotoxicity. Investigative Ophthalmology \& Visual Science, 49, 4515-4522. doi:10.1167/iovs.08-2078

[61] Lam, T.T., Alber, A.S., Kwong, J.M., et al. (1999) N-methyl-D-aspartate (NMDA)-Induced apoptosis in rat retina. Investigative Ophthalmology \& Visual Science, 40, 23912397.

[62] Kuribayashi, J., Kitaoka, Y., Munemasa, Y., et al. (2010) Kinesin-1 and degenerative changes in optic nerve axons in NMDA-Induced neurotoxicity. Brain Research, 1362, 133-140. doi:10.1016/j.brainres.2010.09.053

[63] Michalska-Malecka, K. and Slowinska-Lozynska, L. (2012) Aggregation and deformability of erythrocytes in primary open-angle glaucoma (POAG): The assessment of arterial hypertension. Clinical Hemorheology Microcirculation, 51, 277-285.

[64] Mao, W., Rubin, J.S., Anoruo, N., et al. (2012) SFRP1 promoter methylation and expression in human trabecular meshwork cells. Experimental Eye Research, 97, 130-136. doi:10.1016/j.exer.2012.01.003 